\title{
Lumps, breathers and interaction solutions of a (3+1)-dimensional generalized Kadovtsev-Petviashvili equation
}

\author{
Xi Ma ${ }^{1}$, Tiecheng Xia ${ }^{1}$, and Handong Guo ${ }^{1}$ \\ ${ }^{1}$ Shanghai University
}

May 11, 2020

\begin{abstract}
In this paper, we use the Hirota bilinear method to nd the $\mathrm{N}$-soliton solution of a $(3+1)$-dimensional generalized KadovtsevPetviashvili equation. Then, we obtain the T-order breathers of the equation, and combine the long-wave limit method to give the M-order lumps. Resorting to the extended homoclinic test technique, we obtain the breather-kink solutions for the equation. Last, the interaction solution composed of the K-soliton solution, T-breathers and M-lumps for the (3+1)-dimensional generalized KP equation is constructed.
\end{abstract}

\section{Hosted file}

a 3+1 dimensional generalized KP equation.pdf available at https://authorea.com/users/ 320248/articles/449841-lumps-breathers-and-interaction-solutions-of-a-3-1-dimensionalgeneralized-kadovtsev-petviashvili-equation 
figures/1-SOLITON/1-SOLITON-eps-converted-to.pdf 
figures/2-SOLITON/2-SOLITON-eps-converted-to.pdf 
figures/3-SOLITON/3-SOLITON-eps-converted-to.pdf 
figures/1-SOLITONt/1-SOLITONt-eps-converted-to.pdf 
figures/2-SOLITONt/2-SOLITONt-eps-converted-to.pdf 
figures/3-SOLITONt/3-SOLITONt-eps-converted-to.pdf 
figures/1a-breather/1a-breather-eps-converted-to.pdf 
figures/1b-breather/1b-breather-eps-converted-to.pdf 
figures/1c-breather/1c-breather-eps-converted-to.pdf 
figures/2t-2-breather/2t-2-breather-eps-converted-to.pdf 
figures/2t0-breather/2t0-breather-eps-converted-to.pdf 
figures/2t2-breather/2t2-breather-eps-converted-to.pdf 
figures/2-2t-breather/2-2t-breather-eps-converted-to.pdf 
figures/20t-breather/20t-breather-eps-converted-to.pdf 
figures/22t-breather/22t-breather-eps-converted-to.pdf 
figures/-12t-breather/-12t-breather-eps-converted-to.pdf 
figures/02t-breather/02t-breather-eps-converted-to.pdf 
figures/12t-breather/12t-breather-eps-converted-to.pdf 
figures/31-breather/31-breather-eps-converted-to.pdf 
figures/32-breather/32-breather-eps-converted-to.pdf 
figures/33-breather/33-breather-eps-converted-to.pdf 
figures/breather-kink/breather-kink-eps-converted-to.pdf 
figures/r-breather-kink/r-breather-kink-eps-converted-to.pdf 
figures/t-51-lump/t-51-lump-eps-converted-to.pdf 
figures/t01-lump/t01-lump-eps-converted-to.pdf 
figures/t51-lump/t51-lump-eps-converted-to.pdf 
figures/t-51-lumpc/t-51-lumpc-eps-converted-to.pdf 
figures/t01-lumpc/t01-lumpc-eps-converted-to.pdf 
figures/t51-lumpc/t51-lumpc-eps-converted-to.pdf 
figures/t-32-lump/t-32-lump-eps-converted-to.pdf 
figures/t02-lump/t02-lump-eps-converted-to.pdf 
figures/t32-lump/t32-lump-eps-converted-to.pdf 
figures/-3t2-lumpc/-3t2-lumpc-eps-converted-to.pdf 
figures/0t2-lumpc/0t2-lumpc-eps-converted-to.pdf 
figures/3t2-lumpc/3t2-lumpc-eps-converted-to.pdf 
figures/t-103-lump/t-103-lump-eps-converted-to.pdf 
figures/t03-lump/t03-lump-eps-converted-to.pdf 
figures/t103-lump/t103-lump-eps-converted-to.pdf 
figures/t-103-lumpc/t-103-lumpc-eps-converted-to.pdf 
figures/t03-lumpc/t03-lumpc-eps-converted-to.pdf 
figures/t103-lumpc/t103-lumpc-eps-converted-to.pdf 


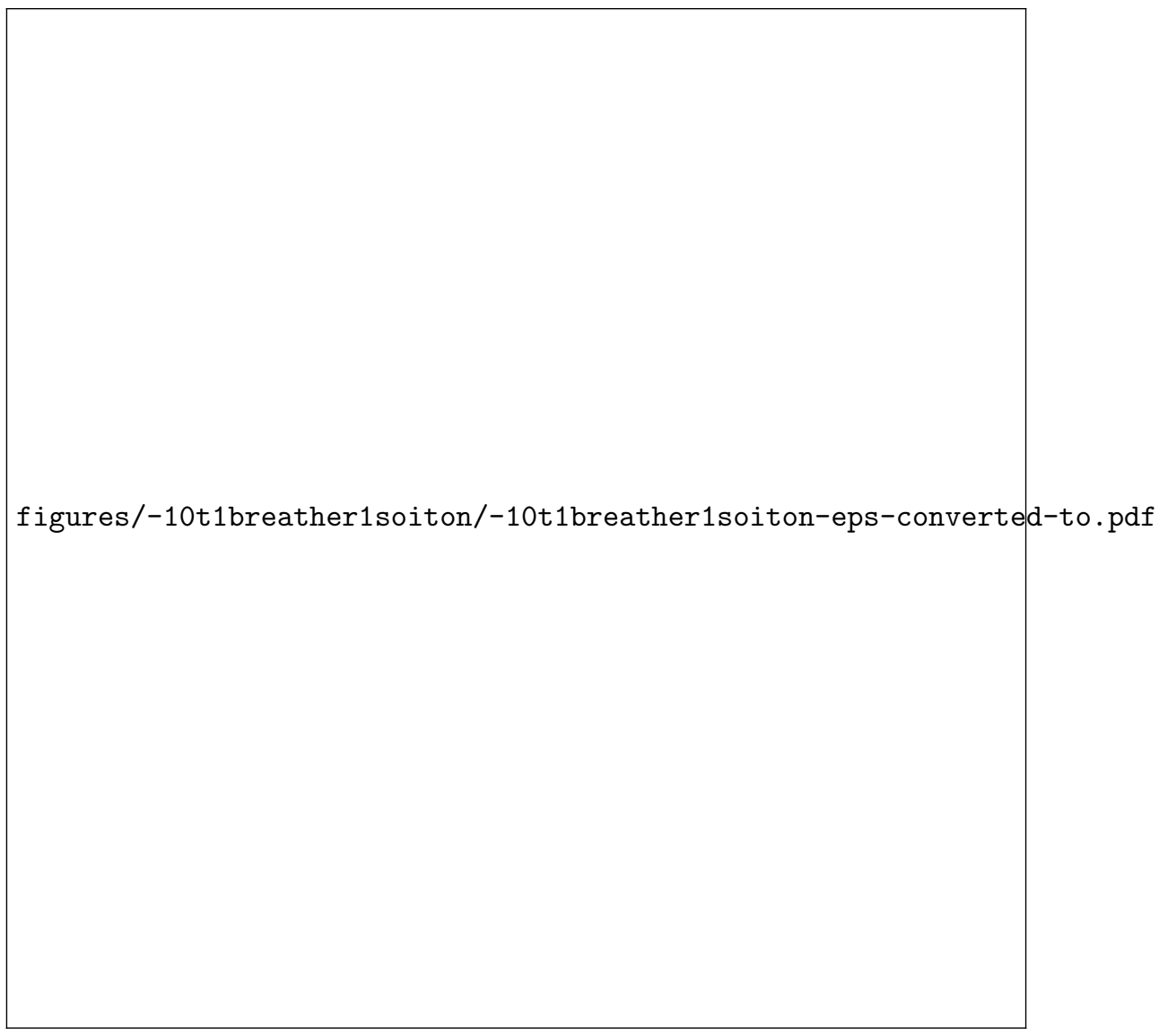




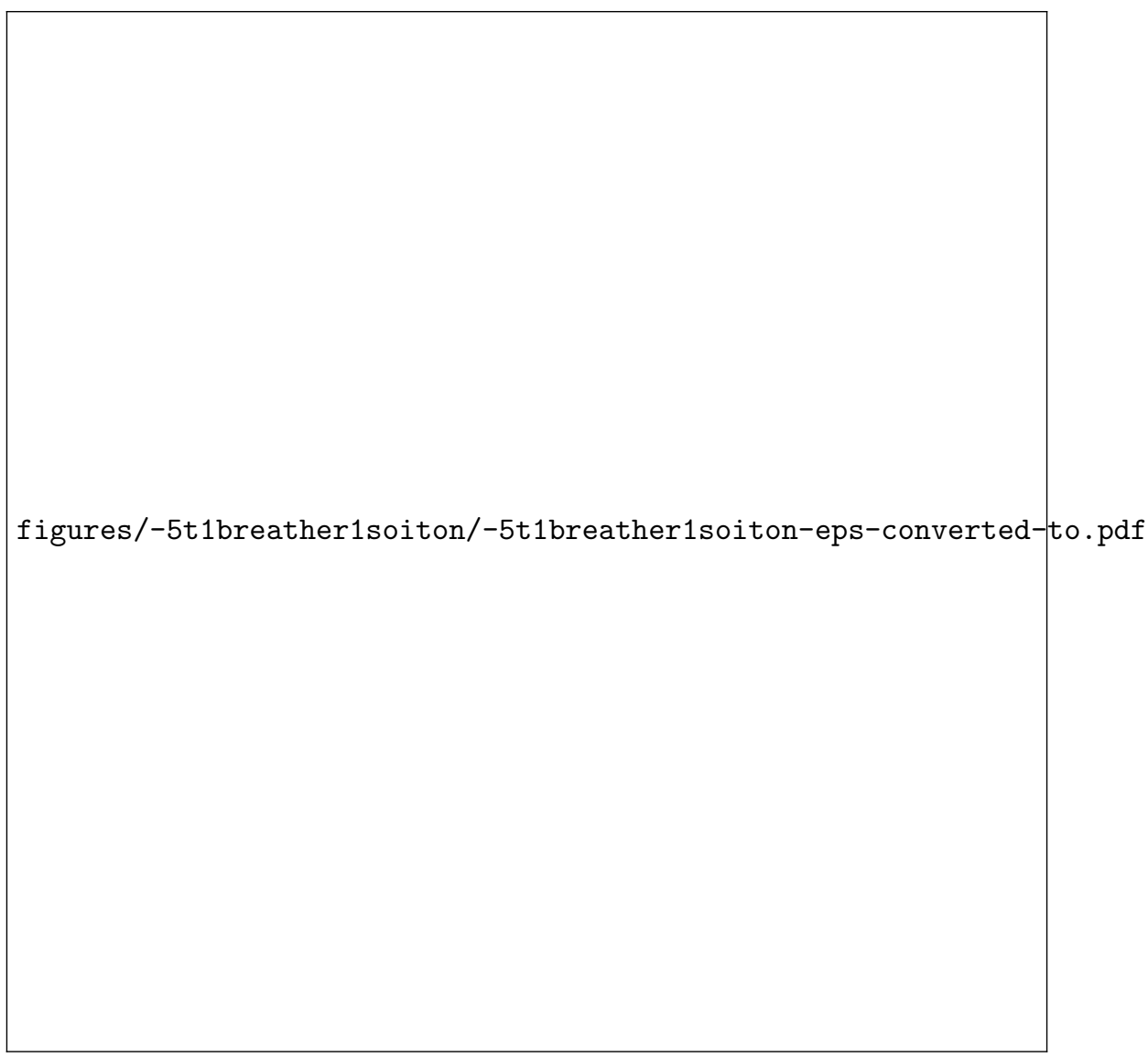




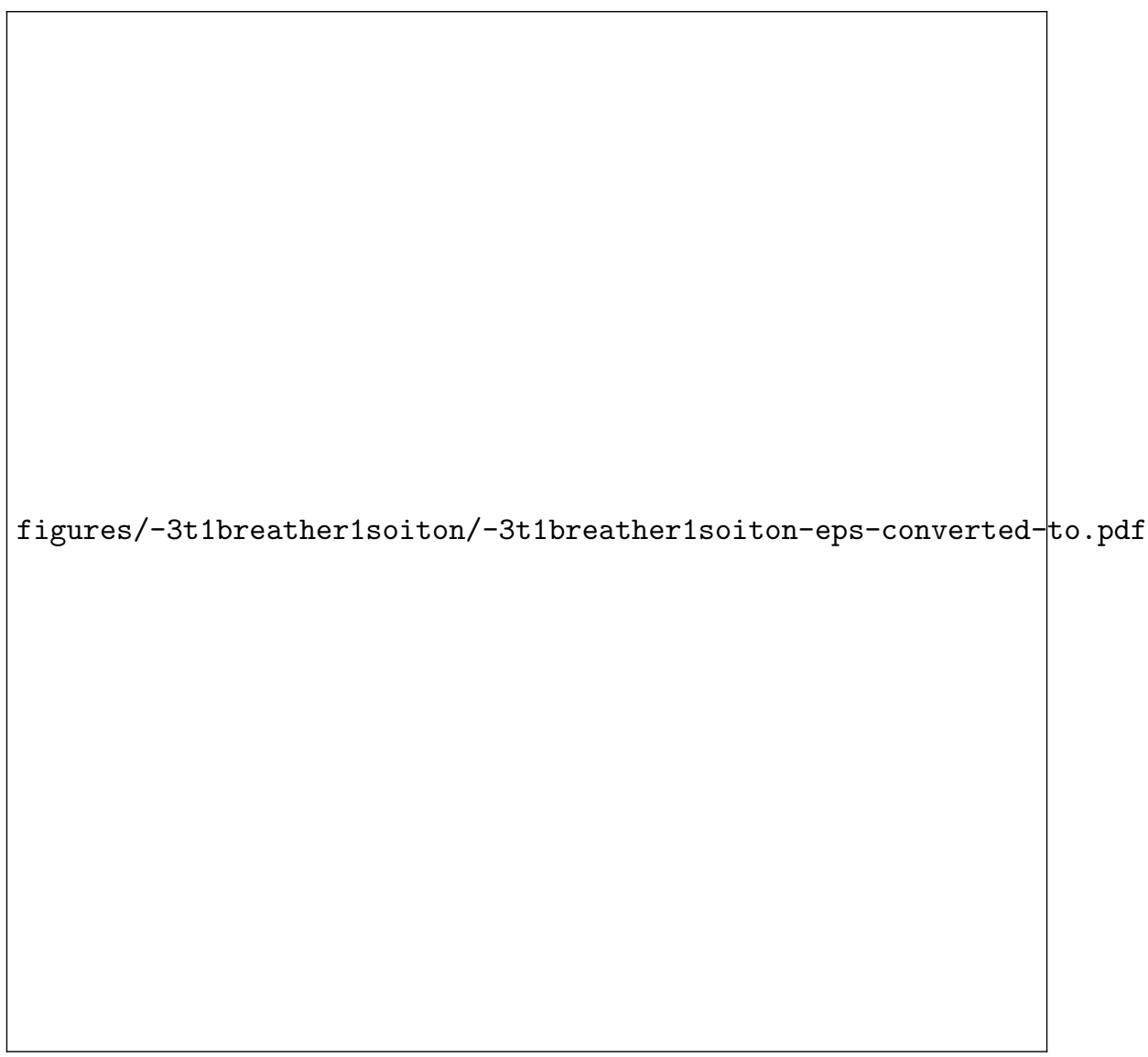




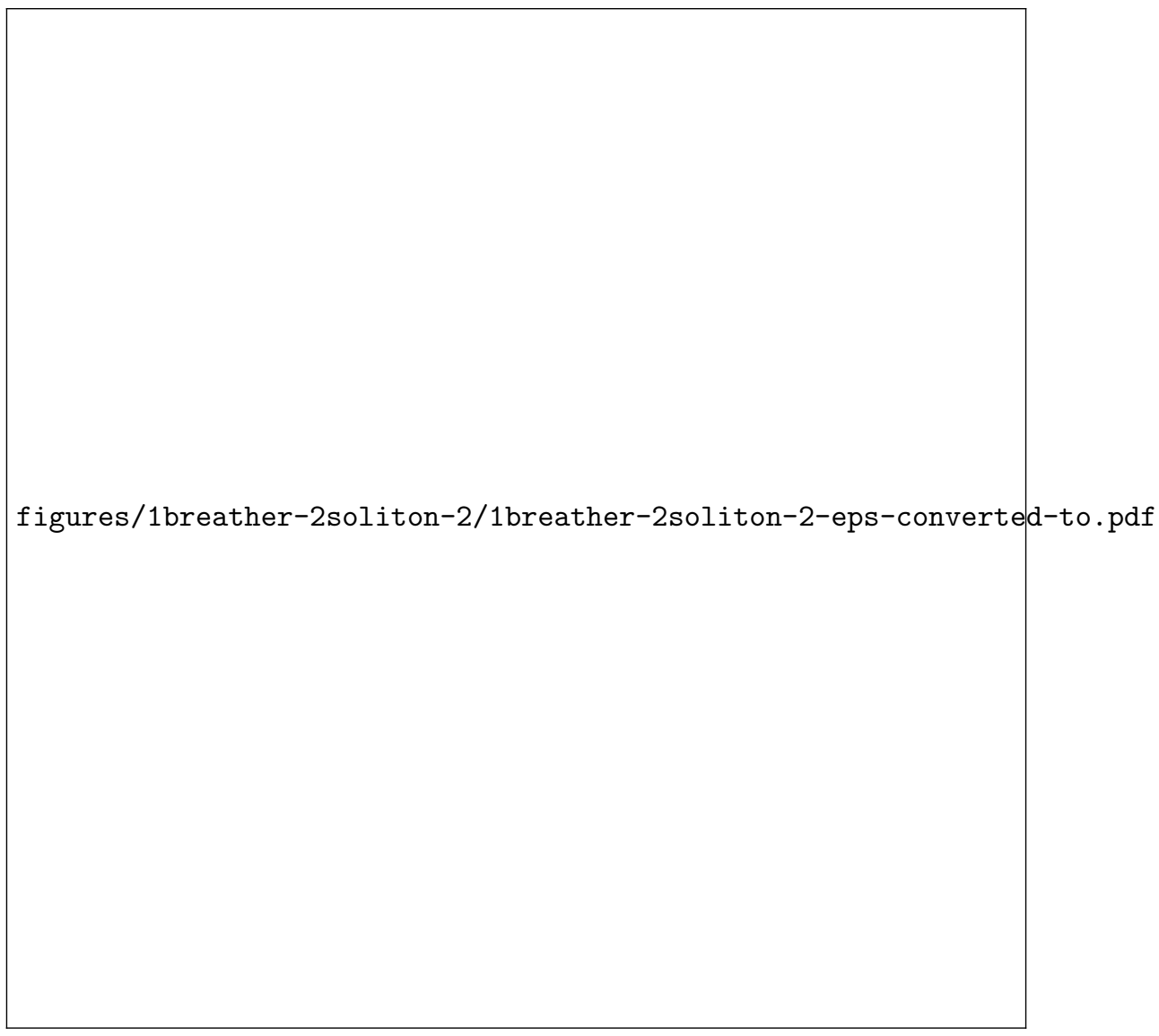




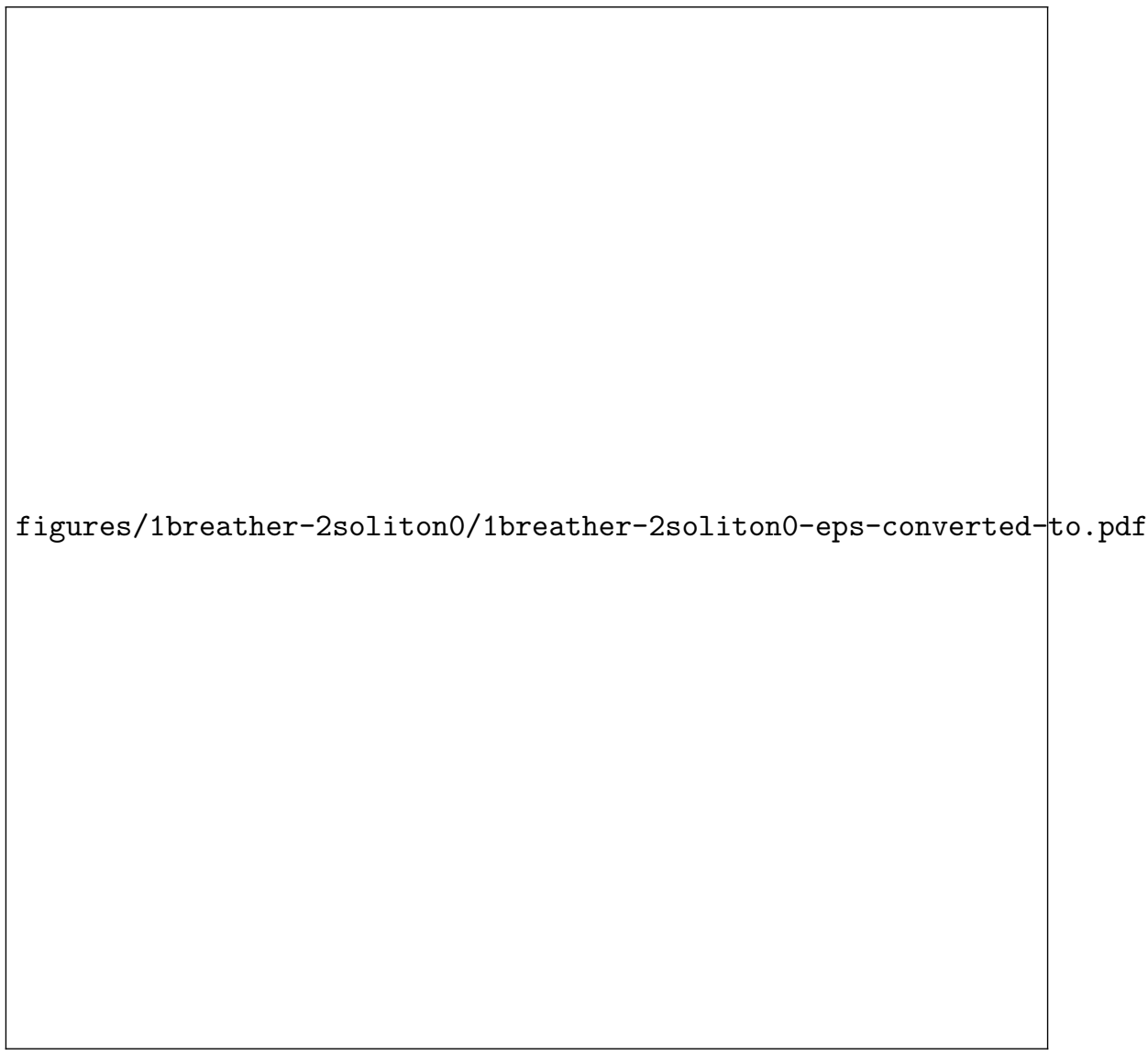




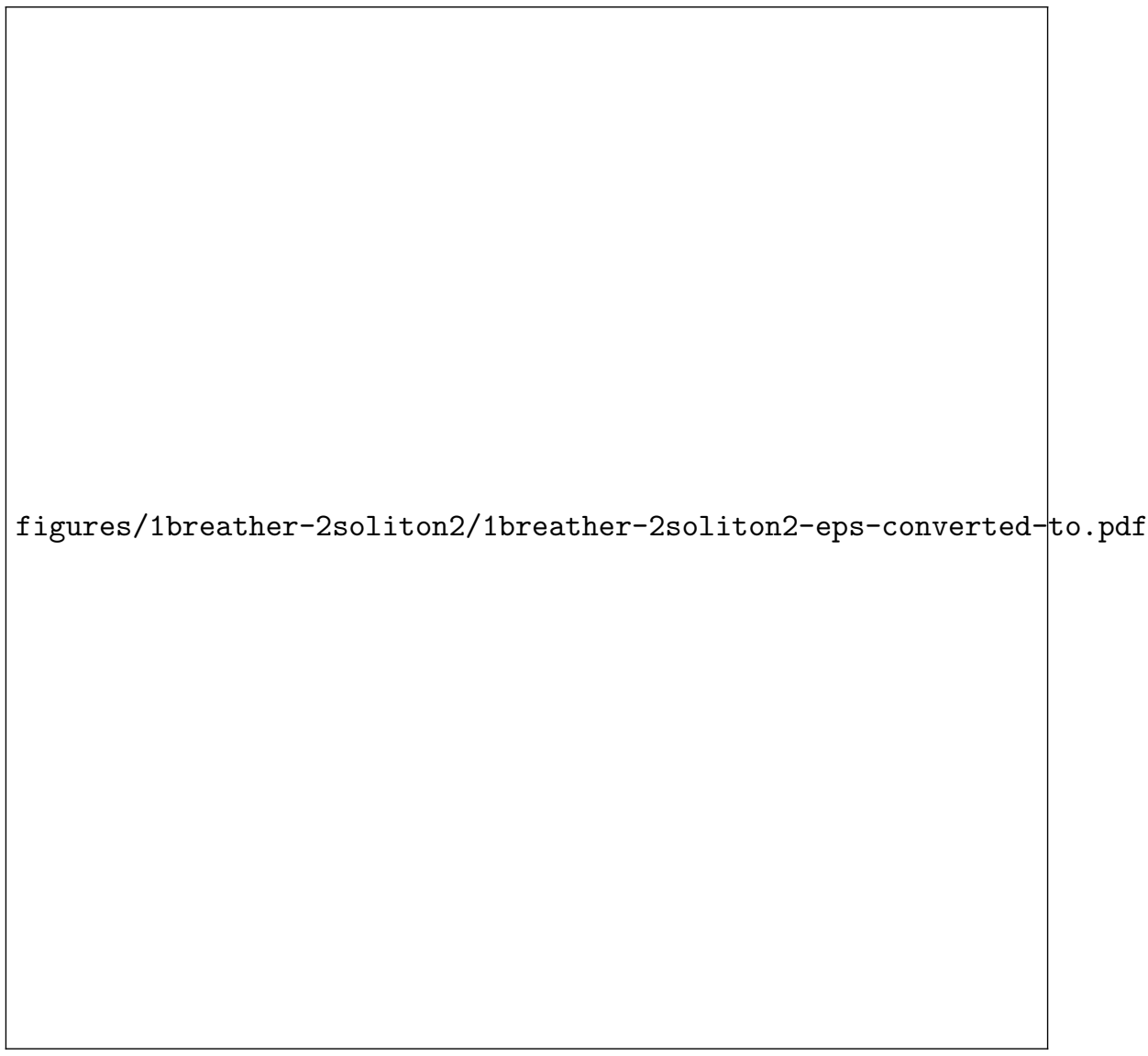


figures/-4t1lump2soliton/-4t1lump2soliton-eps-converted-to.pdf 
figures/0t1lump2soliton/0t1lump2soliton-eps-converted-to.pdf 
figures/4t1lump2soliton/4t1lump2soliton-eps-converted-to.pdf 